\title{
A BDDC Preconditioner for Saddle Point Problems
}

\author{
Jing $\mathrm{Li}^{1}$ and Olof Widlund ${ }^{2}$ \\ 1 Department of Mathematical Sciences, Kent State University, Kent, OH 44242 \\ li@math.kent.edu \\ 2 Courant Institute of Mathematical Sciences, New York University, 251 Mercer \\ Street, New York, NY 10012 widlund@cs.nyu.edu
}

\begin{abstract}
Summary. The purpose of this paper is to extend the BDDC (balancing domain decomposition by constraints) algorithm to saddle point problems that arise when mixed finite element methods are used to approximate the system of incompressible Stokes equations. The BDDC algorithms are defined in terms of a set of primal continuity constraints, which are enforced across the interface between the subdomains, and which provide a coarse space component of the preconditioner. Sets of such constraints are identified for which bounds on the rate of convergence can be established that are just as strong as previously known bounds for the elliptic case. The preconditioned operator is positive definite and a conjugate gradient method can be used. A close connection is also established between the BDDC and FETI-DP algorithms for the Stokes case.
\end{abstract}

\section{Introduction}

The BDDC algorithms are domain decomposition methods based on nonoverlapping subdomains into which the domain of a given partial differential equation is divided. Introduced by Dohrmann [1] and analyzed in the elliptic case by him, Mandel, and Tezaur [9], these methods represent an important advance over the balancing Neumann-Neumann methods that have been used extensively in the past to solve large finite element problems; cf. [10, Section 6.2 ] where references to earlier work can also be found. It has also been established that the preconditioned operators of a pair of BDDC and FETI-DP algorithms, with the same primal constraints, have the same nonzero eigenvalues for positive definite elliptic problems; see $[9,3,7]$.

In this paper, a BDDC preconditioner is developed for mixed finite element approximations of the incompressible Stokes equations in a very similar way; see also [8] for many more details. If the set of primal constraints on the velocity across the interface satisfies a certain assumption, we are then able to show that the preconditioned operator is positive definite and has the same nonzero eigenvalues as the FETI-DP operator developed in [6]. With an 
additional assumption, a bound on the convergence rate as strong as for the standard elliptic case can be proved.

\section{Discretization of a Saddle Point Problem}

Let us consider the incompressible Stokes problem on a bounded, polyhedral domain $\Omega$, in two or three dimensions. We denote the boundary of the domain by $\partial \Omega$; for simplicity a homogeneous Dirichlet boundary condition is enforced. The weak solution has the following saddle point formulation: find $\mathbf{u} \in\left(H_{0}^{1}(\Omega)\right)^{d}=\left\{\mathbf{v} \in\left(H^{1}(\Omega)\right)^{d} \mid \mathbf{v}=\mathbf{0}\right.$ on $\left.\partial \Omega\right\}, d=2$ or 3 , and $p \in L_{0}^{2}(\Omega)=\left\{q \in L^{2}(\Omega) \mid \int_{\Omega} q=0\right\}$, such that,

$$
\left\{\begin{aligned}
a(\mathbf{u}, \mathbf{v})+b(\mathbf{v}, p)=(\mathbf{f}, \mathbf{v}), & \forall \mathbf{v} \in\left(H_{0}^{1}(\Omega)\right)^{d}, \\
b(\mathbf{u}, q) & =0, \quad \forall q \in L_{0}^{2}(\Omega),
\end{aligned}\right.
$$

where $a(\mathbf{u}, \mathbf{v})=\int_{\Omega} \nabla \mathbf{u}: \nabla \mathbf{v}$, or $a(\mathbf{u}, \mathbf{v})=2 \int_{\Omega} \epsilon(\mathbf{u}): \epsilon(\mathbf{v})$ and $b(\mathbf{u}, q)=$ $-\int_{\Omega}(\nabla \cdot \mathbf{u}) q$. Here the strain tensor $\epsilon(\mathbf{u})$ is defined by $\epsilon_{i j}(\mathbf{u})=\left(\frac{\partial u_{i}}{\partial x_{j}}+\frac{\partial u_{j}}{\partial x_{i}}\right) / 2$. The operator form of the Stokes problem with Dirichlet boundary conditions is the same for either choice of the bilinear form $a(\cdot, \cdot)$, but we adopt the second which gives rise to a natural boundary condition which is consistent with physics.

In our mixed finite element methods for solving the saddle point problem (1), the velocity solution space will be denoted by $\widehat{\mathbf{W}}$. It consists of vectorvalued, low order piece-wise polynomial functions which are continuous across element boundaries. The pressure space $Q \subset L_{0}^{2}(\Omega)$ consists of scalar, discontinuous functions. A characteristic diameter of the elements of the underlying triangulation is denoted by $h$. The finite element approximation $(\mathbf{u}, p)$ of the variational problem (1) can be written as

$$
\left[\begin{array}{cc}
A & B^{T} \\
B & 0
\end{array}\right]\left[\begin{array}{l}
\mathbf{u} \\
p
\end{array}\right]=\left[\begin{array}{l}
\mathbf{f} \\
0
\end{array}\right]
$$

We will always assume that the chosen mixed finite element space $\widehat{\mathbf{W}} \times Q$ is inf-sup stable, i.e., that there exists a positive constant $\beta$, independent of $h$, such that

$$
\sup _{\mathbf{w} \in \widehat{\mathbf{W}}} \frac{b(\mathbf{w}, q)}{\|\mathbf{w}\|_{H^{1}}} \geq \beta\|q\|_{L^{2}}, \quad \forall q \in Q .
$$

The domain $\Omega$ is decomposed into $N$ nonoverlapping polyhedral subdomains $\Omega_{i}, i=1,2, \ldots, N$, of characteristic diameter $H$. The subdomain interface is defined by $\Gamma=\left(\cup \partial \Omega_{i}\right) \backslash \partial \Omega$, and the interface of an individual subdomain $\Omega_{i}$ is $\Gamma_{i}=\partial \Omega_{i} \cap \Gamma$. We decompose the discrete velocity and pressure spaces $\widehat{\mathbf{W}}$ and $Q$ into $\widehat{\mathbf{W}}=\mathbf{W}_{I} \oplus \widehat{\mathbf{W}}_{\Gamma}$ and $Q=Q_{I} \oplus Q_{0}$, where $\mathbf{W}_{I}$ 
and $Q_{I}$ are direct sums of subdomain interior velocity spaces $\mathbf{W}_{I}^{(i)}$, and subdomain interior pressure spaces $Q_{I}^{(i)}$, respectively. The elements of $\mathbf{W}_{I}^{(i)}$ are supported in the subdomain $\Omega_{i}$ and vanish on its interface $\Gamma_{i}$, while the elements of $Q_{I}^{(i)}$ are restrictions of elements in $Q$ to $\Omega_{i}$ which satisfy $\int_{\Omega_{i}} q_{I}^{(i)}=0$. $\widehat{\mathbf{W}}_{\Gamma}$ is the space of traces on $\Gamma$ of functions in $\widehat{\mathbf{W}}$ and $Q_{0}$ is the subspace of $Q$ with constant values $q_{0}^{(i)}$ in the subdomain $\Omega_{i}$ that satisfy $\int_{\Omega} q_{0} d x=$ $\sum_{i=1}^{N} q_{0}^{(i)} m\left(\Omega_{i}\right)=0$, where $m\left(\Omega_{i}\right)$ is the measure of the subdomain $\Omega_{i}$.

We denote the space of interface velocity variables on the subdomain $\Omega_{i}$ by $\mathbf{W}_{\Gamma}^{(i)}$, and the associated product space by $\mathbf{W}_{\Gamma}=\prod_{i=1}^{N} \mathbf{W}_{\Gamma}^{(i)}$; generally functions in $\mathbf{W}_{\Gamma}$ are discontinuous across the interface. Eliminating the independent subdomain interior variables $\left(\mathbf{u}_{I}, p_{I}\right)$ from the global problem (2), we have the global interface problem

$$
\left[\begin{array}{cc}
\widehat{S}_{\Gamma} & \widehat{B}_{0 \Gamma}^{T} \\
\widehat{B}_{0 \Gamma} & 0
\end{array}\right]\left[\begin{array}{c}
\mathbf{u}_{\Gamma} \\
p_{0}
\end{array}\right]=\left[\begin{array}{c}
\mathbf{g}_{\Gamma} \\
0
\end{array}\right] .
$$

Here, $\mathbf{g}_{\Gamma}$ is a reduced load vector obtained when the interior variables are

eliminated. $\widehat{S}_{\Gamma}$ is assembled from subdomain Stokes Schur complements

$$
S_{\Gamma}^{(i)}=A_{\Gamma \Gamma}^{(i)}-\left[\begin{array}{ll}
A_{\Gamma I}^{(i)} & B_{I \Gamma}^{(i)^{T}}
\end{array}\right]\left[\begin{array}{cc}
A_{I I}^{(i)} & B_{I I}^{(i)^{T}} \\
B_{I I}^{(i)} & 0
\end{array}\right]^{-1}\left[\begin{array}{c}
A_{\Gamma I}^{(i)^{T}} \\
B_{I \Gamma}^{(i)}
\end{array}\right],
$$

i.e., $\widehat{S}_{\Gamma}=\sum_{i=1}^{N} R_{\Gamma}^{(i)^{T}} S_{\Gamma}^{(i)} R_{\Gamma}^{(i)}$, where $R_{\Gamma}^{(i)}$ is the operator which maps functions in the continuous interface velocity space $\widehat{\mathbf{W}}_{\Gamma}$ to their subdomain components in the space $\mathbf{W}_{\Gamma}^{(i)}$. Denote by $S_{\Gamma}$ and $R_{\Gamma}$ the direct sums of $S_{\Gamma}^{(i)}$ and $R_{\Gamma}^{(i)}$, respectively. $\widehat{S}_{\Gamma}$ can then be written as $\widehat{S}_{\Gamma}=R_{\Gamma}^{T} S_{\Gamma} R_{\Gamma}$.

We denote the operator of the interface problem (4) by $\widehat{S}$. Since $\widehat{S}$ is symmetric and indefinite, we could use the minimal residual method, possibly with a positive definite block preconditioner, as in [10, Section 9.2], to solve problem (4). We will instead propose a different type of preconditioner and show that the preconditioned operator is positive definite, provided that a suitable set of primal constraints are chosen; cf. Assumption 1.

\section{A BDDC Preconditioner for Stokes Equations}

We introduce a partially assembled interface velocity space $\widetilde{\mathbf{W}}_{\Gamma}$ by

$$
\widetilde{\mathbf{W}}_{\Gamma}=\widehat{\mathbf{W}}_{\Pi} \bigoplus \mathbf{w}_{\Delta}=\widehat{\mathbf{W}}_{\Pi} \bigoplus\left(\prod_{i=1}^{N} \mathbf{w}_{\Delta}^{(i)}\right) .
$$

Here, $\widehat{\mathbf{W}}_{\Pi}$ is the continuous coarse level, primal interface velocity space which is typically spanned by subdomain vertex nodal basis functions, and/or by interface edge and/or face basis functions with constant values, or with values 
of weight functions, on these edges or faces. These basis functions correspond to the primal interface velocity continuity constraints. We will always assume that the basis has been changed so that each primal basis function corresponds to an explicit degree of freedom which is shared by the neighboring subdomains; see [7], [5, Section 6], and [4] for more details of the change of basis. The complimentary space $\mathbf{W}_{\Delta}$ is the direct sum of the subdomain dual interface velocity spaces $\mathbf{W}_{\Delta}^{(i)}$, which correspond to the remaining interface velocity degrees of freedom and are spanned by basis functions which vanish at the primal degrees of freedom. Thus, an element in the space $\widetilde{\mathbf{W}}_{\Gamma}$ has a continuous primal velocity and typically a discontinuous dual velocity component.

We define $R_{\Delta}^{(i)}$ as the operator which maps a function in the space $\widehat{\mathbf{W}}_{\Gamma}$ to its dual component in the space $\mathbf{W}_{\Delta}^{(i)} \cdot R_{\Gamma \Pi}$ is the restriction operator from the space $\widehat{\mathbf{W}}_{\Gamma}$ to its subspace $\widehat{\mathbf{W}}_{\Pi}$ and $R_{\Pi}^{(i)}$ is the operator which maps $\widehat{\mathbf{W}}_{\Pi}$ into its $\Gamma_{i}$-component. $\widetilde{R}_{\Gamma}$ is the direct sum of $R_{\Gamma \Pi}$ and the $R_{\Delta}^{(i)}$, and it is a map from $\widehat{\mathbf{W}}_{\Gamma}$ into $\widetilde{\mathbf{W}}_{\Gamma}$.

The interface velocity Schur complement $\widetilde{S}_{\Gamma}$ is defined on the partially assembled interface velocity space $\widetilde{\mathbf{W}}_{\Gamma}$ by $\widetilde{S}_{\Gamma}=\bar{R}_{\Gamma}^{T} S_{\Gamma} \bar{R}_{\Gamma}$, where $\bar{R}_{\Gamma}$ maps $\widetilde{\mathbf{W}}_{\Gamma}$ into the product space $\mathbf{W}_{\Gamma}$ associated with the set of subdomains. We recall that the global interface Schur operator $\widehat{S}_{\Gamma}$ is obtained by fully assembling the $S_{\Gamma}^{(i)}$ across the subdomain interface. $\widehat{S}_{\Gamma}$ can therefore also be obtained from $\widetilde{S}_{\Gamma}$ by further assembling the dual interface velocity part, i.e., $\widehat{S}_{\Gamma}=\widetilde{R}_{\Gamma}^{T} \widetilde{S}_{\Gamma} \widetilde{R}_{\Gamma}$. Correspondingly, we define $\widetilde{B}_{0 \Gamma}$, which is obtained from the subdomain operators $B_{0 \Gamma}^{(i)}$ by assembling the primal interface velocity part only. The operator $\widehat{B}_{0 \Gamma}$ can then be obtained from $\widetilde{B}_{0 \Gamma}$ by assembling the dual interface velocity part on the subdomain interfaces, i.e., $\widehat{B}_{0 \Gamma}=\widetilde{B}_{0 \Gamma} \widetilde{R}_{\Gamma}$. We can therefore write $\widehat{S}$, the operator of the global interface problem (4), as $\widehat{S}=\widetilde{R} T \widetilde{S} \widetilde{R}$, where

$$
\widetilde{R}=\left[\begin{array}{cc}
\widetilde{R}_{\Gamma} & \\
& I
\end{array}\right], \quad \widetilde{S}=\left[\begin{array}{cc}
\widetilde{S}_{\Gamma} & \widetilde{B}_{0 \Gamma}^{T} \\
\widetilde{B}_{0 \Gamma} & 0
\end{array}\right] .
$$

To define the BDDC preconditioner, we need certain scaling functions. For each interface node $x \in \Gamma_{i}$, we set $\delta_{i}^{\dagger}(x)=1 / \mathcal{N}_{x}, x \in \Gamma_{i}$, where $\mathcal{N}_{x}$ is the number of subdomain to which $x$ belongs. Given the scaling factors at the subdomain interface nodes, we can define scaled restriction operators $R_{D, \Delta}^{(i)}$. Each row of $R_{\Delta}^{(i)}$ has only one nonzero entry which corresponds to a node $x \in \Gamma_{i}$, and multiplying each such element with the scaling factor $\delta_{i}^{\dagger}(x)$ gives us $R_{D, \Delta}^{(i)}$. The scaled operator $\widetilde{R}_{D, \Gamma}$ is the direct sum of $R_{\Gamma \Pi}$ and the $R_{D, \Delta}^{(i)}$. For elasticity problems, these scaling factors should depend on the first Lamé constant $\mu$, which can be allowed to change across the interface between neighboring subdomains; see [10, Section 8.5.1] and [5].

The BDDC preconditioner for solving the interface saddle point problem (4) is $M^{-1}=\widetilde{R}_{D}^{T} \widetilde{S}^{-1} \widetilde{R}_{D}$, where $\widetilde{R}_{D}$ is of the same form as $\widetilde{R}$ in (5), except 
that $\widetilde{R}_{\Gamma}$ is replaced by $\widetilde{R}_{D, \Gamma}$. To compute the product of $\widetilde{S}^{-1}$ and a vector, a coarse level saddle point problem, for the primal variables, and subdomain Neumann problems, each with a few primal constraints, need to be solved; cf. $[7,8]$.

\section{Condition Number Bounds}

We define an average operator

$$
E_{D}=\widetilde{R} \widetilde{R}_{D}^{T}=\left[\begin{array}{rr}
\widetilde{R}_{\Gamma} & \\
& I
\end{array}\right]\left[\begin{array}{cc}
\widetilde{R}_{D, \Gamma}^{T} & \\
& I
\end{array}\right]=\left[\begin{array}{lll}
E_{D, \Gamma} & \\
& & I
\end{array}\right],
$$

which maps $\widetilde{\mathbf{W}}_{\Gamma} \times Q_{0}$, with generally discontinuous interface velocities, to elements with continuous interface velocities in the same space. $E_{D, \Gamma}=\widetilde{R}_{\Gamma} \widetilde{R}_{D, \Gamma}^{T}$, provides the average of the interface velocities across the interface $\Gamma$. Denoting the primal and dual parts of $\mathbf{w}_{\Gamma}$ by $\mathbf{w}_{\Pi}$ and $\mathbf{w}_{\Delta}$, we can write $E_{D, \Gamma} \mathbf{w}_{\Gamma}$ as the direct sum of $\mathbf{w}_{\Pi}$ and $E_{D, \Delta} \mathbf{w}_{\Delta}$, where $E_{D, \Delta} \mathbf{w}_{\Delta}$ is the dual part of the averaged vector.

The following two assumptions will be needed in the condition number bound of the preconditioned operator.

Assumption 1 For any $\mathbf{w}_{\Delta} \in \mathbf{W}_{\Delta}, \int_{\partial \Omega_{i}} \mathbf{w}_{\Delta}^{(i)} \cdot \mathbf{n}=0$ and $\int_{\partial \Omega_{i}}\left(E_{D, \Delta} \mathbf{w}_{\Delta}\right)^{(i)}$. $\mathbf{n}=0$, where $\mathbf{n}$ is the unit outward normal of $\partial \Omega_{i}$.

Assumption 2 There exists a positive constant $C$, which is independent of $H, h$, and the number of subdomains, such that

$$
\left|\bar{R}_{\Gamma}\left(E_{D, \Gamma} \mathbf{w}_{\Gamma}\right)\right|_{\mathbf{E}(\Gamma)} \leq C\left(1+\log \frac{H}{h}\right)\left|\bar{R}_{\Gamma} \mathbf{w}_{\Gamma}\right|_{\mathbf{E}(\Gamma)}, \quad \forall \mathbf{w}_{\Gamma} \in \widetilde{\mathbf{W}}_{\Gamma},
$$

where $|\cdot|_{\mathbf{E}(\Gamma)}$ is defined on the space $\mathbf{W}_{\Gamma}$ by $\left|\mathbf{w}_{\Gamma}\right|_{\mathbf{E}(\Gamma)}^{2}=\sum_{i=1}^{N}\left|\mathbf{w}_{\Gamma}^{(i)}\right|_{\mathbf{E}\left(\Gamma_{i}\right)}^{2}$ with

$$
\left|\mathbf{w}_{\Gamma}^{(i)}\right| \mathbf{E}\left(\Gamma_{i}\right)=\inf _{\substack{\mathbf{v}^{(i)} \in\left(\left.H^{1}\left(\Omega_{i}\right) d \\ \mathbf{v}^{(i)}\right|_{\Gamma_{i}}=\mathbf{w}_{\Gamma}^{(i)}\right.}}\left\|\epsilon\left(\mathbf{v}^{(i)}\right)\right\|_{L^{2}\left(\Omega_{i}\right)} .
$$

These two assumptions can be satisfied with an appropriate choice of the primal continuity constraints on the interface velocity variables; for twodimensional problems, Assumptions 1 and 2 are satisfied if all subdomain vertices are primal, i.e, both components of the velocity are continuous at those nodes, and $\int_{\Gamma^{i j}} \mathbf{w}_{\Gamma}^{(i)} \cdot \mathbf{n}_{i j}=\int_{\Gamma^{i j}} \mathbf{w}_{\Gamma}^{(j)} \cdot \mathbf{n}_{i j}$, is enforced on all the subdomain interface edges. Here $\mathbf{n}_{i j}$ is a normal of $\Gamma_{i j}$. For the more complicated three-dimensional case, see $[2,8,5]$.

The interface velocity subspaces $\widehat{\mathbf{W}}_{\Gamma, B}$ and $\widetilde{\mathbf{W}}_{\Gamma, B}$ are defined by $\widehat{\mathbf{W}}_{\Gamma, B}=$ $\left\{\mathbf{w}_{\Gamma} \in \widehat{\mathbf{W}}_{\Gamma} \mid \widehat{B}_{0 \Gamma} \mathbf{w}_{\Gamma}=0\right\}$, and $\widetilde{\mathbf{W}}_{\Gamma, B}=\left\{\mathbf{w}_{\Gamma} \in \widetilde{\mathbf{W}}_{\Gamma} \mid \widetilde{B}_{0 \Gamma} \mathbf{w}_{\Gamma}=0\right\}$. We 
Jing Li and Olof Widlund

will call $\widehat{\mathbf{W}}_{\Gamma, B} \times Q_{0}$ and $\widetilde{\mathbf{W}}_{\Gamma, B} \times Q_{0}$ the benign subspaces of $\widehat{\mathbf{W}}_{\Gamma} \times Q_{0}$ and $\widetilde{\mathbf{W}}_{\Gamma} \times Q_{0}$, respectively.

The preconditioned operator $\widetilde{R}_{D}^{T} \widetilde{S}^{-1} \widetilde{R}_{D} \widehat{S}$ is indefinite on the space $\widehat{\mathbf{W}}_{\Gamma} \times$ $Q_{0}$, since both $\widehat{S}$ and $\widetilde{S}$ are indefinite. However, both $\widehat{S}$ and $\widetilde{S}$ are positive semidefinite, when restricted to the benign subspaces $\widehat{\mathbf{W}}_{\Gamma, B} \times Q_{0}$ and $\widetilde{\mathbf{W}}_{\Gamma, B} \times Q_{0}$, respectively. We will also know, from Lemma 1 , that $M^{-1} \widehat{S}$ maps $\widehat{\mathbf{W}}_{\Gamma, B} \times Q_{0}$ into itself and that $M^{-1} \widehat{S}$ is symmetric with respect to the bilinear form $\langle\cdot, \cdot\rangle_{\widehat{S}}$. Theorem 1 will show that $M^{-1} \widehat{S}$ is positive definite, when restricted to the benign subspace $\widehat{\mathbf{W}}_{\Gamma, B} \times Q_{0}$. Therefore a preconditioned conjugate gradient method can be used. The following lemmas will be used in the proof of Theorem 1.

Lemma 1 Let Assumption 1 hold. Then, $\widetilde{R}_{D}^{T} \mathbf{w} \in \widehat{\mathbf{W}}_{\Gamma, B} \times Q_{0}$, for any $\mathbf{w} \in$ $\widetilde{\mathbf{W}}_{\Gamma, B} \times Q_{0}$.

Lemma 2 Let Assumptions 1 and 2 hold. There then exists a positive constant $C$, which is independent of $H, h$, and the number of subdomains, such that,

$$
<E_{D} \mathbf{w}, E_{D} \mathbf{w}>_{\widetilde{S}} \leq C \frac{1}{\beta^{2}}\left(1+\log \frac{H}{h}\right)^{2}<\mathbf{w}, \mathbf{w}>_{\widetilde{S}}, \quad \forall \mathbf{w} \in \widetilde{\mathbf{W}}_{\Gamma, B} \times Q_{0} .
$$

Here, $\beta$ is the inf-sup stability constant of Equation (3).

Theorem 1 Let Assumptions 1 and 2 hold. The preconditioned operator $M^{-1} \widehat{S}$ is then symmetric, positive definite with respect to the bilinear form $\langle\cdot, \cdot\rangle_{\widehat{S}}$ on the benign space $\widehat{\mathbf{W}}_{\Gamma, B} \times Q_{0}$. Its minimum eigenvalue is 1 and its maximum eigenvalue is bounded by

$$
C \frac{1}{\beta^{2}}\left(1+\log \frac{H}{h}\right)^{2} .
$$

Here, $C$ is a constant which is independent of $H, h$, and the number of subdomains and $\beta$ is the inf-sup stability constant defined in Equation (3).

Just as in the positive definite elliptic case, we can also establish that the preconditioned BDDC operator and the preconditioned FETI-DP operator in [6] have the same nonzero eigenvalues; cf. [7, 8]. We have,

Theorem 2 Let Assumption 1 hold. The preconditioned FETI-DP and BDDC operators have the same nonzero eigenvalues, when the same set of primal constraints are applied.

\section{Numerical Experiments}

We solve a lid-driven-cavity problem on the domain $\Omega=[0,1] \times[0,1]$ with Dirichlet boundary condition, where the velocity is $(1,0)$ on the upper side, 
and vanishes on the other three sides. We use a uniform mesh, as in Figure 1. The mixed finite element method is also indicated in Figure 1; the velocity is continuous and linear in each element and the pressure is constant on macro elements which are unions of four triangles.
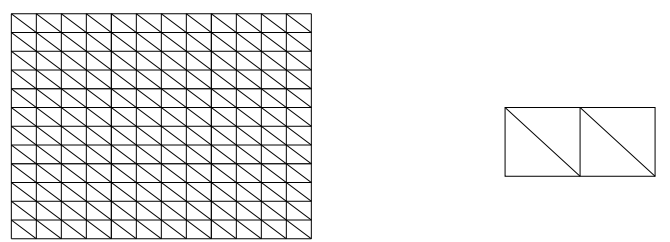

Fig. 1. The mesh and the mixed finite elements.

Both the BDDC and FETI-DP algorithms have been tested. The preconditioned conjugate gradient method is used and the iteration is halted when the $L_{2}$-norm of the residual has been reduced by a factor $10^{-6}$. The primal velocity space is spanned by the subdomain vertex nodal basis functions for both components and by a constant vector in the direction normal to the edge for each interface edge. Both Assumptions 1 and 2 are then satisfied. From Tables 1 and 2, we see that the preconditioned BDDC and FETI-DP operators are both positive definite and quite well-conditioned as established in Theorems 1 and 2. We observe that the extreme eigenvalues and the iteration counts of the BDDC and FETI-DP algorithms match very well, and that the condition numbers of both algorithms are independent of the number of subdomains, and increases only slowly with the number of elements across each subdomain, all as predicted by the theory.

Table 1. Spectral bounds and iteration counts for a pair of BDDC and FETI-DP algorithms, with different number of subdomains, for $H / h=8$ and a primal space spanned by both corner and normal edge basis functions.

\begin{tabular}{|c||c|c|c||c|c|c|}
\hline \multicolumn{1}{|c||}{ Num. of subs } & \multicolumn{3}{c||}{ BDDC } & \multicolumn{3}{c|}{ FETI-DP } \\
\cline { 2 - 7 }$n_{x} \times n_{y}$ & $\lambda_{\min }$ & $\lambda_{\max }$ & iter. & $\lambda_{\min }$ & $\lambda_{\max }$ & iter. \\
\hline $4 \times 4$ & 1.00 & 3.14 & 11 & 1.00 & 3.14 & 11 \\
$8 \times 8$ & 1.00 & 3.88 & 12 & 1.00 & 3.88 & 12 \\
$12 \times 12$ & 1.00 & 4.02 & 12 & 1.00 & 4.02 & 13 \\
$16 \times 16$ & 1.00 & 4.06 & 12 & 1.00 & 4.07 & 13 \\
$20 \times 20$ & 1.00 & 4.08 & 12 & 1.00 & 4.08 & 13 \\
\hline
\end{tabular}

When Assumption 1 is not satisfied, e.g., when only vertex velocity variables are primal, the preconditioned BDDC operator is no longer positive definite, and the iteration counts will depend on both the number of subdomains as well as on the number of elements across each subdomain; cf. [8]. 
Table 2. Spectral bounds and iteration counts for a pair of BDDC and FETI-DP algorithms, with different $H / h$, for $4 \times 4$ subdomains and a primal space spanned by both corner and normal edge basis functions.

\begin{tabular}{|c||c|c|c||c|c|c|}
\hline \multicolumn{1}{|c||}{} & \multicolumn{3}{c||}{ BDDC } & \multicolumn{3}{c|}{ FETI-DP } \\
\cline { 2 - 7 } & $\lambda_{\min }$ & $\lambda_{\max }$ & iter. & $\lambda_{\min }$ & $\lambda_{\max }$ & iter. \\
\hline 4 & 1.00 & 2.17 & 8 & 1.00 & 2.17 & 9 \\
8 & 1.00 & 3.14 & 11 & 1.00 & 3.14 & 11 \\
16 & 1.00 & 4.22 & 13 & 1.00 & 4.22 & 12 \\
32 & 1.00 & 5.42 & 14 & 1.00 & 5.42 & 14 \\
\hline
\end{tabular}

\section{References}

1. Clark R. Dohrmann. A preconditioner for substructuring based on constrained energy minimization. SIAM J. Sci Comput., 25(1):246-258, 2003.

2. Clark R. Dohrmann. A substructuring preconditioner for nearly incompressible elasticity problems. Technical report, Sandia National Laboratories, Albuquerque, New Mexico, October 2004.

3. Yannis Fragakis and Manolis Papadrakakis. The mosaic of high performance domain decomposition methods for structural mechanics: Formulation, interrelation and numerical efficiency of primal and dual methods. Comput. Methods Appl. Mech. Engrg, 192(35-36):3799-3830, 2003.

4. Axel Klawonn and Oliver Rheinbach. A parallel implementation of Dual-Primal FETI methods for three dimensional linear elasticity using a transformation of basis. Technical Report SM-E-601, University of Duisburg-Essen, February 2005.

5. Axel Klawonn and Olof B. Widlund. Dual-Primal FETI methods for linear elasticity. Technical Report TR2004-855, Department of Computer Science, Courant Institute, September 2004.

6. Jing Li. A dual-primal FETI method for incompressible Stokes equations. Technical report, Courant Institute of Mathematical Sciences, 2001. TR-816, Department of Computer Science.

7. Jing Li and Olof B. Widlund. FETI-DP, BDDC, and Block Cholesky Methods. Technical report, Courant Institute of Mathematical Sciences, December 2004. TR2004-857, Department of Computer Science. Submitted to Internat. J. Numer. Methods Engrg.

8. Jing $\mathrm{Li}$ and Olof B. Widlund. BDDC algorithms for incompressible Stokes equations. Technical Report TR2005-861, Department of Computer Science, Courant Institute, April 2005.

9. Jan Mandel, Clark R. Dohrmann, and Radek Tezaur. An algebraic theory for primal and dual substructuring methods by constraints. Appl. Numer. Math., 2005. To appear.

10. Andrea Toselli and Olof B. Widlund. Domain Decomposition Methods - Algorithms and Theory, volume 34 of Springer Series in Computational Mathematics. Springer Verlag, Berlin-Heidelberg-New York, 2004. 